\title{
Another Interpretation of Diversity Gain of MIMO Systems
}

\author{
Shuichi Ohno ${ }^{1}$ and Kok Ann Donny Teo ${ }^{2}$ \\ ${ }^{1}$ Hiroshima University \\ ${ }^{2}$ DSO National Laboratories \\ ${ }^{1}$ Japan \\ ${ }^{2}$ Singapore
}

\section{Introduction}

Multiple-Input Multiple-Output (or the so-called MIMO) system, which employs multiple antennas at both ends of the receiver and transmitter terminals, has been the subject of intensive research efforts in the past decade with potential application in high speed wireless communications network. This is chiefly motivated by the benefits of 1) the spatial multiplexing gain, which makes use of the degrees of freedom in communication system by transmitting independent symbol streams in parallel through spatial channels, to improve bandwidth efficiency; 2) diversity gain, which can be achieved by averaging performance over multiple path gains to combat fading, to improve channel capacity and/or bit-error rate (BER). Information theoretical analysis reveals that MIMO systems indeed offer high spectral efficiency (Foschini, 1996; Goldsmith et al., 2003; Telatar, 1999). It has been shown in (Tse and Viswanath, 2005) that the capacity of an $N_{r} \times N_{t}$ MIMO system with $N_{t}$ transmit and $N_{r}$ receive antennas over i.i.d. Rayleigh fading channels scales with the minimum of the number $N_{t}$ of transmit antennas and the number $N_{r}$ of receive antennas at the high SNR regime. With ideal capacity achieving Gaussian codes, capacity is attained by minimum mean squared error successive interference cancellation (MMSE-SIC) at the receiver (Tse and Viswanath, 2005) if the number of receive antennas is equal to or larger than the number of transmit antennas. The receive diversity achieved by endorsing multiple receive antennas have been utilized in practical communication systems. Recently, Space-Time codes have also been developed to obtain transmit antenna diversity gain (Alamouti, 1998; Caire and Shamai, 1999; Ma and Giannakis, 2003; Tarokh et al., 1999; Xin et al., 2003). Performance gains induced by different schemes of MIMO systems were comprehensively compared in (Catreux et al., 2003).

It is well-known that there is a tradeoff between multiplexing gain and diversity gain. The diversity gain is usually measured by the slope of the BER curve. Over i.i.d. Rayleigh distributed channels, the diversity order of $N_{r} \times N_{t}$ systems with linear equalization is given by $N_{r}-N_{t}+1$ at high SNR at full multiplexing (Winters et al., 1994). This implies that given a fixed number $N_{t}$ of transmit antennas, increasing the number $N_{r}$ of receive antennas increases the diversity order. Conversely, given a fixed $N_{r}$, an increase in $N_{t}$ (which contributes to multiplexing gain) decreases the diversity order. In (Narasimhan, 2003), by exploiting the 
tradeoff, an adaptive control of the number of transmit antennas and symbol constellations is proposed to improve the performance of spatial multiplexing in correlated fading channels. Moreover, theoretical analysis that shows a fundamental tradeoff between multiplexing gain and/or diversity gain including Vertical-Bell Laboratories Layered Space-Time (V-BLAST) and Space-Time Codes (STC) have been reported (Tse and Viswanath, 2005; Zheng and Tse, 2003).

Capacity or ergodic capacity, which is the capacity averaged over fading channels, are often utilized to evaluate capacity gain. On the other hand, BER or average BER, which is the BER averaged over fading channels, relate to diversity gain. These gains have been analyzed by approximate expressions for these measures at the SNR extremes, or by directly evaluating them for a particular channel probability density function (pdf), e.g., i.i.d. complex-normal distribution (Chiani et al., 2003; Marzetta and Hochwald, 1999; Smith et al., 2003). However, since the full diversity order appears only at high SNR, having higher diversity order does not necessarily mean having better performance at a particular value of SNR. Moreover, diversity gain of Rayleigh channels does not necessarily imply the existence of diversity gain for other distributed channels. In this chapter, we study universal properties of the performance of MIMO system as in (Ohno and Teo, 2007), which is independent of channel probability density functions and hold at any SNR.

We only consider the case where the performance measure is a convex or concave function of SNR. However, it is shown that important performance measures, including channel capacity and BER, are convex or concave. Thus, our results are significant. To get more insights into MIMO systems, we study capacity gain from a different point of view. A similar approach is adopted in (Ohno and Teo, 2007) to analyze the impact of antenna size of MIMO systems on BER performance with zero-forcing (ZF) equalization.

Take channel capacity for example. Let us suppose that you can install an additional receive antenna in the $N_{r} \times N_{t}$ system to construct an $\left(N_{r}+1\right) \times N_{t}$ system. Assume that the underlying channel environment is not time-varying (i.e., static). Then, can any other gain (besides power gain) be obtained by increasing the number of receive antennas? Without the values of channel coefficients or the associated channel pdf, no one can answer this question or evaluate the possible gain correctly. Now, we look at the problem from another perspective. For simplicity, we put $N_{r}=2$ and $N_{t}=2$. From a $3 \times 2$ system, we can remove one receive antenna in three different ways to obtain three possible $2 \times 2$ systems. Then, we compare the performance of the original $3 \times 2$ system with the average performance of the three $2 \times 2$ systems. We show in this chapter that without the knowledge of channel coefficients and at any value of $\mathrm{SNR}$, the capacity of the original $3 \times 2$ system is greater than the average capacity of the three $2 \times 2$ systems. More generally, our analysis reveals that increasing the number of receive antennas generates capacity gain even in static channels. From this, we can prove that the mean capacity with respect to channel pdf, which is mathematically equivalent to the so-called ergodic capacity for fading channels, increases as the number of receive antennas increases at any value of SNR. Our proof relies not on the channel pdf but on the concavity of the capacity function. This implies that the concavity is indispensable to obtain receive antenna diversity.

Next, we consider removing a transmit antenna from an $N_{r} \times N_{t}$ system and compare the capacity of the $N_{r} \times N_{t}$ system with the average capacity of $N_{r} \times\left(N_{t}-1\right)$ systems. Clearly, removing one transmit antenna reduces the multiplexing gain. For comparison, we adopt the capacity per transmit antenna as a parameter. Then, we prove that reducing the number 
of transmit antennas improves the capacity per transmit antenna. It follows that the mean capacity per transmit antenna degrades as the number of transmit antennas increases at any value of SNR irrespective of channel pdf. This means that increasing the number of transmit antennas improves the multiplexing gain but degrades the capacity per transmit antenna. There exists a tradeoff between multiplexing gain and capacity gain regardless of channel pdf and SNR.

Although we do not evaluate how much gains there actually are, which requires the knowledge of channel coefficients or channel pdf, our results are universal in the sense that performance ordering with the number of transmit antennas and the number of receive antenna is independent of channel pdf and holds true at any value of SNR. We also study the achievable information rate of block minimum mean squared error (MMSE) equalization to obtain similar results.

\section{Preliminaries and system model}

We consider a MIMO transmission with $N_{t}$ transmit and $N_{r}$ receive antennas over flat non-frequency-selective channels. Let us define $\rho / N_{t}$ as the transmit power at each transmit antenna for the $N_{r} \times N_{t}$ MIMO system. We denote the path gain from transmit antenna $n$ $\left(n \in\left[1, N_{t}\right]\right)$ to receive antenna $m\left(m \in\left[1, N_{r}\right]\right)$ as $h_{m n}$. The path gains are assumed to be unknown to the transmitter but perfectly known to the receiver.

Let the received signal at receive antenna $m$ be $x_{m}$. The $N_{r}$ received signals are arranged in a vector as $\boldsymbol{x}=\left[x_{1}, \ldots, x_{N_{r}}\right]^{T}$, where $[\cdot]^{T}$ denotes transposition. Then, $\boldsymbol{x}$ is expressed as

$$
\boldsymbol{x}=\sqrt{\frac{\rho}{N_{t}}} \boldsymbol{H} \boldsymbol{s}+\boldsymbol{w},
$$

where the $N_{r} \times N_{t}$ channel matrix $\boldsymbol{H}$, the $N_{t} \times 1$ combined data vector $\boldsymbol{s}$ having i.i.d. entries with unit variance, the $N_{r} \times 1$ vector $\boldsymbol{w}$ of zero mean circular complex additive white Gaussian noise (AWGN) entries with unit variance are respectively given by

$$
\begin{gathered}
\boldsymbol{H}=\left[\begin{array}{ccc}
h_{11} & \ldots & h_{1 N_{t}} \\
\vdots & \ddots & \vdots \\
h_{N_{r} 1} & \ldots & h_{N_{r} N_{t}}
\end{array}\right], \\
\boldsymbol{s}=\left[\begin{array}{lll}
s_{1} & \ldots & s_{N_{t}}
\end{array}\right]^{T}, \\
\boldsymbol{w}=\left[\begin{array}{lll}
w_{1} & \ldots & w_{N_{r}}
\end{array}\right]^{T} .
\end{gathered}
$$

Let the $m$ th row (which corresponds to the $m$ th receive antenna) of the channel matrix $\boldsymbol{H}$ be $\boldsymbol{h}_{m}$ for $m \in\left[1, N_{r}\right]$, and the $n$th column (which corresponds to the $n$th transmit antenna) of the channel matrix $\boldsymbol{H}$ be $\tilde{\boldsymbol{h}}_{n}$ for $n \in\left[1, N_{t}\right]$ so that we can also express the channel matrix as

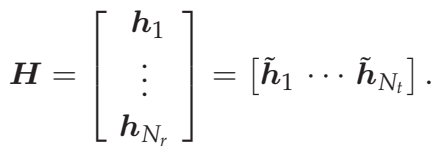

The signal-to-noise ratio (SNR) at receive antenna $m$ is found to be $\rho\left\|\boldsymbol{h}_{m}\right\|^{2} / N_{t}$, where $\|\cdot\|$ is the 2-norm of a vector, while the overall receive power of the symbol transmitted from antenna $n$, i.e., the sum of power from transmit antenna $n$ at all receive antennas, is $\rho\left\|\tilde{\boldsymbol{h}}_{n}\right\|^{2} / N_{t}$. 
With capacity achieving Gaussian codes, for a given channel $\boldsymbol{H}$, the information rate of the $N_{r} \times N_{t}$ MIMO system is expressed as (see. e.g. (Telatar, 1999; Tse and Viswanath, 2005))

$$
\mathcal{C}_{N_{r}, N_{t}}=\log \left|\boldsymbol{I}_{N_{r}}+\frac{\rho}{N_{t}} \boldsymbol{H} \boldsymbol{H}^{\mathcal{H}}\right|=\log \left|\boldsymbol{I}_{N_{t}}+\frac{\rho}{N_{t}} \boldsymbol{H}^{\mathcal{H}} \boldsymbol{H}\right|,
$$

where $(\cdot)^{\mathcal{H}}$ stands for complex conjugate transposition. Over fading channels, MIMO system offers the benefits of multiplexing gain and/or capacity/diversity gain (Larsson and Stoica, 2003; Tse and Viswanath, 2005).

For our analysis that follows, we utilize the achievable information rates of non-linear Maximum Likelihood (ML) equalization and minimum mean squared error (MMSE) equalization. MMSE equalizations at the receiver becomes available if the channel matrix has column full rank, which requires $N_{r} \geq N_{t}$.

Let us shortly review MMSE equalization for MIMO systems. If we employ block-by-block equalization, the MMSE equalizer is given by $\boldsymbol{G}=\sqrt{\frac{\rho}{N_{t}}} \boldsymbol{H}^{\mathcal{H}}\left(\frac{\rho}{N_{t}} \boldsymbol{H} \boldsymbol{H}^{\mathcal{H}}+\boldsymbol{I}_{N_{r}}\right)^{-1}$. The equalized output is thus expressed as $\hat{\mathbf{s}}=\mathbf{G} \boldsymbol{x}$. We define the $n$th entry of the equalized output as $\hat{s}_{n}=p_{n} s_{n}+v_{n}$, where $v_{n}$ is the effective noise contaminating the $n$th symbol. Then, we can show that the signal-to-interference noise ratio (SINR) of symbol $n$ after MMSE equalization is expressed as (Kay, 1993; Tse and Viswanath, 2005)

$$
\operatorname{SINR}_{N_{r}, N_{t}, n}=\frac{\rho}{N_{t}} \tilde{\boldsymbol{h}}_{n}^{\mathcal{H}}\left(\boldsymbol{I}_{N_{r}}+\frac{\rho}{N_{t}} \sum_{l=1, l \neq n}^{N_{t}} \tilde{\boldsymbol{h}}_{l} \tilde{\boldsymbol{h}}_{l}^{\mathcal{H}}\right)^{-1} \tilde{\boldsymbol{h}}_{n} .
$$

Block-by-block MMSE equalization can be easily implemented but cannot achieve the capacity except for some special cases. Capacity is achieved by MMSE successive interference cancellation (MMSE-SIC) at the receiver. Then, SIC with optimal cancellation order is utilized in Vertical-Bell Laboratories Layered Space-Time (V-BLAST) (Foschini et al., 1999). Although cancellation order affects the BER performance, it does not change the achievable information rate (Tse and Viswanath, 2005, Chapter 8). Thus, it is convenient in what follows to only consider the simplest MMSE-SIC that does not perform the optimal ordering (i.e., arbitrary ordering) procedure. We first equalize symbols from transmit antenna 1 . Then after decoding them, the contribution of the signal due to the symbol from transmit antenna 1 is reconstructed and eradicated from the received vector. The same procedure is repeated for the remaining symbols from transmit antenna 2 to transmit antenna $N_{t}$. If we denote the SINR of the equalized output at the $n$th step of MMSE-SIC as $\operatorname{SINR}_{n}^{S I C}$ and there is no error propagation, then the capacity in (6) can be adequately expressed as (Tse and Viswanath, 2005, Chapter 8)

$$
\mathcal{C}_{N_{r}, N_{t}}=\sum_{n=1}^{N_{t}} \log \left(1+\operatorname{SINR}_{n}^{S I C}\right) \text {. }
$$

\section{Decreasing the number of receive antennas}

Based on the mathematical tools in the previous section, we investigate information rates of MIMO systems when we decrease the number of receive antennas, while fixing the number of transmit antennas. As the number of receive antennas decreases/increases, the overall receive power decreases/increases, which is known as power loss/gain. Thus, it seems obvious that capacity degrades as the number of receive antennas decreases. However, the MIMO system 
may have different receive power from each transmit antenna and the same conclusion is not self-evident. This begs the question: Given the "fair" condition that the overall receive power from each transmit antenna is kept constant even if the number of receive antennas decreases, does capacity decreases or increases? We study how the capacity is affected by the number of receive antennas when the overall receive power from each transmit antenna is fixed.

Let us define a sample correlation of the channel matrix $\boldsymbol{H}$ as

$$
\boldsymbol{R}_{N_{r}, N_{t}}=\boldsymbol{H}^{\mathcal{H}} \boldsymbol{H}=\sum_{m=1}^{N_{r}} \boldsymbol{h}_{m}^{\mathcal{H}} \boldsymbol{h}_{m} .
$$

Assuming that $N_{r} \geq 2$, we fix the number of transmit antennas at $N_{t}$ and decrease the number $N_{r}$ of receive antennas by one. When receive antenna $\mu$ is removed from the $N_{r} \times N_{t}$ system, the corresponding channel matrix is denoted as $\boldsymbol{H}^{(\mu)}$. The $\left(N_{r}-1\right) \times N_{t}$ channel matrix $\boldsymbol{H}^{(\mu)}$ yields the $N_{t} \times N_{t}$ correlation matrix $\boldsymbol{R}_{N_{r}-1, N_{t}}^{(\mu)}$ corresponding to (9), expressed as

$$
\boldsymbol{R}_{N_{r}-1, N_{t}}^{(\mu)}=\boldsymbol{H}^{(\mu) \mathcal{H}} \boldsymbol{H}^{(\mu)}=\sum_{m=1, m \neq \mu}^{N_{r}} \boldsymbol{h}_{m}^{\mathcal{H}} \boldsymbol{h}_{m} .
$$

It is easy to see that the matrices $\boldsymbol{R}_{N_{r}, N_{t}}$ and $\boldsymbol{R}_{N_{r}-1, N_{t}}^{(\mu)}$ are related as

$$
\sum_{\mu=1}^{N_{r}} \boldsymbol{R}_{N_{r}-1, N_{t}}^{(\mu)}=\left(N_{r}-1\right) \boldsymbol{R}_{N_{r}, N_{t}} .
$$

If we remove one receive antenna from the $N_{r} \times N_{t}$ system, there are $N_{r}$ possible systems having $N_{r}-1$ receive antennas. We compare the capacity of the $N_{r} \times N_{t}$ system with the average capacity of $\left(N_{r}-1\right) \times N_{t}$ systems with respect to antenna selection. This average capacity is equivalent to the average capacity when we uniformly remove one receive antenna among $N_{r}$ antennas, i.e., the selection of any one receive antenna has the same probability $1 / N_{r}$.

If receive antenna $\mu$ is removed from the $N_{r} \times N_{t}$ system, then the overall receive power from transmit antenna $n$ reduces to $\rho \sum_{m=1, m \neq \mu}^{N_{r}}\left|h_{m n}\right|^{2} / N_{t}$. Thus, for $\left(N_{r}-1\right) \times N_{t}$ system, the average overall receive power from antenna $n$ with respect to random receive antenna dropping is given by

$$
\frac{1}{N_{r}} \sum_{\mu=1}^{N_{r}}\left(\sum_{m=1, m \neq \mu}^{N_{r}} \rho \frac{\left|h_{m n}\right|^{2}}{N_{t}}\right)=\left(\frac{N_{r}-1}{N_{r}}\right) \rho \frac{\left\|\tilde{\boldsymbol{h}}_{n}\right\|^{2}}{N_{t}},
$$

which depicts a reduction in the average overall receive power from antenna $n$. To ensure that the average overall receive power from each transmit antenna remains constant even when the number of receive antennas is reduced by one, we increase the transmit power of the $\left(N_{r}-1\right) \times N_{t}$ system by a factor of $\frac{N_{r}}{N_{r}-1}$, i.e., we replace $\rho$ in (12) by $\frac{N_{r}}{N_{r}-1} \rho$. Then, for this $\left(N_{r}-1\right) \times N_{t}$ system, the receive SNR at receive antenna $m$ increases to $\frac{N_{r}}{N_{r}-1} \frac{\rho\left\|\boldsymbol{h}_{m}\right\|^{2}}{N_{t}}$ and hence the average overall receive power of the $\left(N_{r}-1\right) \times N_{t}$ systems is equal to the overall receive power of the $N_{r} \times N_{t}$ system. Thus, the effects of power loss due to the reduction of the number of receive antennas disappears on the average. 
The information rate of the $\left(N_{r}-1\right) \times N_{t}$ system without receive antenna $\mu$ is expressed as

$$
\mathcal{C}_{N_{r}-1, N_{t}}^{(\mu)}=\log \left|\boldsymbol{I}_{N_{t}}+\left(\frac{N_{r}}{N_{r}-1} \frac{\rho}{N_{t}}\right) \boldsymbol{R}_{N_{r}-1, N_{t}}^{(\mu)}\right|
$$

Then, we have from (11) that

$$
\frac{\rho}{N_{t}} \boldsymbol{R}_{N_{r}, N_{t}}=\frac{1}{N_{r}} \sum_{\mu=1}^{N_{r}}\left(\frac{N_{r}}{N_{r}-1} \frac{\rho}{N_{t}}\right) \boldsymbol{R}_{N_{r}-1, N_{t}}^{(\mu)} .
$$

At this stage, we utilize a fundamental property of log det function: its concavity property. Since log det is a concave function in positive definite matrices, substituting (14) into (6), we find that

$$
\begin{aligned}
\mathcal{C}_{N_{r}, N_{t}} & =\log \left|\boldsymbol{I}_{N_{t}}+\frac{1}{N_{r}} \sum_{\mu=1}^{N_{r}}\left(\frac{N_{r}}{N_{r}-1} \frac{\rho}{N_{t}}\right) \boldsymbol{R}_{N_{r}-1, N_{t}}^{(\mu)}\right| \\
& \geq \frac{1}{N_{r}} \sum_{\mu=1}^{N_{r}} \log \left|\boldsymbol{I}_{N_{t}}+\left(\frac{N_{r}}{N_{r}-1} \frac{\rho}{N_{t}}\right) \boldsymbol{R}_{N_{r}-1, N_{t}}^{(\mu)}\right|=\frac{1}{N_{r}} \sum_{\mu=1}^{N_{r}} \mathcal{C}_{N_{r}-1, N_{t}}^{(\mu)}
\end{aligned}
$$

where the equality holds if and only if all $\boldsymbol{R}_{N_{r}-1, N_{t}}^{(\mu)}$ for $\mu \in\left[1, N_{r}\right]$ can be diagonalized with the same unitary matrix.

Eq. (15) shows that for a fixed channel, the capacity of the $N_{r} \times N_{t}$ system is not smaller than the average capacity of $\left(N_{r}-1\right) \times N_{t}$ systems taken over antenna dropping. It should be noted that the average is not taken over fading channels. For a static channel, we find another disadvantage/advantage of decreasing/increasing the number of receive antennas in addition to power loss/gain. Indeed, (15) is fundamental, from which we will see later that the mean capacity of MIMO systems is also an increasing function in the number of receive antennas at any value of SNR irrespective of channel pdf. Eq. (15) comes only from the basic property of the log det function. It is worth emphasizing that the capacity gain achieved by increasing the number of receive antennas is a direct consequence of the concavity of the $\log$ det function.

To analyze the average capacity over random channels, let us denote the channel probability density function (pdf) of channel $\boldsymbol{H}$ as $P(\boldsymbol{H})$ and similarly for $\boldsymbol{H}^{(\mu)}$ as $P\left(\boldsymbol{H}^{(\mu)}\right)$. We consider the following channel characteristics:

\section{Assumption 1.}

$$
P\left(\boldsymbol{H}^{(1)}\right)=P\left(\boldsymbol{H}^{(2)}\right)=\cdots=P\left(\boldsymbol{H}^{\left(N_{r}\right)}\right) .
$$

This implies that when any one row is removed from the $N_{r} \times N_{t}$ channel matrix, the resultant $\left(N_{r}-1\right) \times N_{t}$ channel matrix has the same probability density function. Clearly, if the entries of $\boldsymbol{H}$ are i.i.d., then (16) holds true. However, it should be remarked that a more general class of channels which includes for example, non i.i.d. channels having correlation between channel gains, meets (16).

The mean capacity is defined as the expectation of the capacity with respect to channel pdf, i.e.,

$$
E\left\{\mathcal{C}_{N_{r}, N_{t}}\right\}=\int \mathcal{C}_{N_{r}, N_{t}} P(\boldsymbol{H}) d \boldsymbol{H}
$$


where $E\{\cdot\}$ denotes the expectation operator. This is mathematically equivalent to the so-called ergodic capacity if the channel is slowly fading and the channel statistics are ergodic. Consequently, it follows from (15) that

$$
E\left\{\mathcal{C}_{N_{r}, N_{t}}\right\}>\frac{1}{N_{r}} \sum_{\mu=1}^{N_{r}} \int \mathcal{C}_{N_{r}-1, N_{t}}^{(\mu)} P(\boldsymbol{H}) d \boldsymbol{H}=\frac{1}{N_{r}} \sum_{\mu=1}^{N_{r}} E\left\{\mathcal{C}_{N_{r}-1, N_{t}}^{(\mu)}\right\},
$$

where the equality sign is removed since the equality in (15) holds only for some special channel realizations. Under Assumption 1, $E\left\{\mathcal{C}_{N_{r}-1, N_{t}}^{(\mu)}\right\}=E\left\{\mathcal{C}_{N_{r}-1, N_{t}}\right\}$, where $E\left\{\mathcal{C}_{N_{r}-1, N_{t}}\right\}$ is the mean capacity of $\left(N_{r}-1\right) \times N_{t}$ system. Thus, we can conclude that:

Theorem 1. Let the capacity of an $M \times N M I M O$ system be $\mathcal{C}_{M, N}$. If the MIMO channel satisfies Assumption 1, then the average capacity taken over channel pdf is an increasing function in the number of receive antennas, i.e.,

$$
E\left\{\mathcal{C}_{N_{r}, N_{t}}\right\}>E\left\{\mathcal{C}_{N_{r}-1, N_{t}}\right\}
$$

where $N_{r} \times N_{t}$ system and $\left(N_{r}-1\right) \times N_{t}$ system have the same receive power.

Theorem 1 clearly states the capacity gain in MIMO transmission that can be acquired by simply increasing the number of receive antennas. A special case of Theorem 1 is well-known where at high SNR, the diversity order of $N_{r} \times N_{t}$ systems over i.i.d. Rayleigh distributed channels with linear equalization is $N_{r}-N_{t}+1$ at full multiplexing (Winters et al., 1994). Here, no approximation is made and no channel pdf is specified except for Assumption 1 to obtain Theorem 1. It is universal in the sense that (19) holds not just for a specific channel pdf but for all kinds of channel pdf meeting Assumption 1, and at all values of SNR. The capacity gain that arises from increasing the number of receive antennas always exists, since it is a result not attributed to the distinct characteristic of Rayleigh fading but attributed to the basic property of the log det function. Hypothetically, if log det were convex (which is never the case), the inequality in (15) and hence the inequality in (19) would be reverse. Thus, the concavity of the log det function is indispensable to obtain receive antenna diversity. To know how much the actual gain is, one has to evaluate the expectation using the underlying channel pdf. In some special channel pdf, e.g., complex-normal distribution, one could derive an analytical expression of the corresponding capacity gain, e.g., as in (Winters et al., 1994).

\subsection{Block MMSE equalization case}

Assuming that the channel matrix $\boldsymbol{H}$ is tall and has column full rank, let us analyze the achievable information rate with block MMSE equalization.

After block MMSE equalization, we have $N_{t}$ parallel channels. Then, the achievable information rate, denoted as $\mathcal{C}_{N_{r}, N_{t}}^{B}$, of $N_{r} \times N_{t}$ system with block MMSE equalization can be expressed as

$$
\mathcal{C}_{N_{r}, N_{t}}^{B}=\sum_{n=1}^{N_{t}} \log \left(1+\operatorname{SINR}_{N_{r}, N_{t}, n}\right) .
$$

If we define the (post-processing) SINR for symbol $s_{n}$ after block MMSE equalization when receive antenna $\mu$ is removed as $\operatorname{SINR}_{N_{r}-1, N_{t}, n}^{(\mu)}$ for $n \in\left[1, N_{t}\right]$, then the achievable information rate of the $\left(N_{r}-1\right) \times N_{t}$ system is

$$
\mathcal{C}_{N_{r}-1, N_{t}}^{B,(\mu)}=\sum_{n=1}^{N_{t}} \log \left(1+\operatorname{SINR}_{N_{r}-1, N_{t}, n}^{(\mu)}\right) .
$$


We utilize the following inequality in (Ohno and Teo, 2007):

$$
\operatorname{SINR}_{N_{r}, N_{t}, n} \geq \frac{1}{N_{r}} \sum_{\mu=1}^{N_{r}} \operatorname{SINR}_{N_{r}-1, N_{t}, n}^{(\mu)} .
$$

The R.H.S. of (22) denotes the average SINR of symbol $n$ when one receive antenna is randomly dropped.

Since $\log$ is a concave function, we have from (22) that

$$
\mathcal{C}_{N_{r}, N_{t}}^{B} \geq \frac{1}{N_{r}} \sum_{n=1}^{N_{t}} \sum_{\mu=1}^{N_{r}} \log \left(1+\operatorname{SINR}_{N_{r}-1, N_{t}, n}^{(\mu)}\right)=\frac{1}{N_{r}} \sum_{\mu=1}^{N_{r}} \mathcal{C}_{N_{r}-1, N_{t}}^{B,(\mu)} .
$$

This states a deterministic yet universal characteristics of the achievable information rate of MIMO systems with block MMSE equalization. For a given channel environment, if a receive antenna is randomly dropped, the average information rate with respect to random antenna dropping degrades except for some special cases. Indeed, the average information rate depends on the number of receive antennas and a fortiori deteriorates as the number of receive antennas is lessened.

By using a similar derivation of Theorem 1, averaging (23) over channel pdf leads to:

Theorem 2. Let the achievable information rate of an $M \times N$ MIMO system be $\mathcal{C}_{M, N}^{B}$, when block MMSE equalization is adopted. If the MIMO channel satisfies Assumption 1, then the achievable information rate averaged over channel pdf is an increasing function in the number of receive antennas, i.e.,

$$
E\left\{\mathcal{C}_{N_{r}, N_{t}}^{B}\right\}>E\left\{\mathcal{C}_{N_{r}-1, N_{t}}^{B}\right\},
$$

where $N_{r} \times N_{t}$ system and $\left(N_{r}-1\right) \times N_{t}$ system have the same receive power.

Theorem 2 states that capacity gain with increasing number of receive antennas exists even for block MMSE equalization. Theorem 1 as well as Theorem 2 highlight the advantage/disadvantage of MIMO system upon increasing/decreasing the number of receive antennas.

\section{Decreasing the number of transmit antennas}

In this section, we consider the information rate for a fixed number $N_{r}$ of receive antennas when the number $N_{t}$ of transmit antennas is reduced by one, assuming that $2 \leq N_{t} \leq N_{r}$. For comparison between $N_{r} \times N_{t}$ system and $N_{r} \times\left(N_{t}-1\right)$ system, as in the previous section, we uniformly remove one transmit antenna among $N_{t}$ transmit antennas, i.e., the selection of any one transmit antenna has the same probability $1 / N_{t}$.

It is often the case that the sum of total transmit power of all transmit antennas is kept constant for different numbers of transmit antennas. But, here we fix the transmit power of each transmit antenna to be $\rho / N_{t}$. This implies that the sum of transmit power reduces from $\rho$ to $\rho\left(N_{t}-1\right) / N_{t}$, if one transmit antenna is removed. In this case, the overall receive power from a transmit antenna remains constant, while the average receive power at each receive antenna of $N_{r} \times\left(N_{t}-1\right)$ system with respect to antenna dropping is $\left(N_{t}-1\right) / N_{t}$ of the receive power at each receive antenna of the original $N_{r} \times N_{t}$ system. 
For analysis, we recall block MMSE equalization and MMSE-SIC equalization. If there is no error propagation in SIC, then we have

$$
\operatorname{SINR}_{N_{r}, N_{t}, n} \leq \operatorname{SINR}_{n}^{\text {SIC }},
$$

where $\operatorname{SINR}_{N_{r}, N_{t}, n}$ and $\operatorname{SINR}_{n}^{S I C}$ are respectively the SINR of symbol from transmit antenna $n$ in block MMSE equalization and in MMSE-SIC equalization. For all $n \in\left[1, N_{t}\right]$, the equalities in (25) hold if and only if the channel matrix $\boldsymbol{H}$ has orthogonal columns.

We can decompose the capacity of $N_{r} \times N_{t}$ system by the following manipulation:

$$
\begin{aligned}
\mathcal{C}_{N_{r}, N_{t}} & =\log \left|\boldsymbol{I}_{N_{r}}+\frac{\rho}{N_{t}} \sum_{n=1}^{N_{t}} \tilde{\boldsymbol{h}}_{n} \tilde{\boldsymbol{h}}_{n}^{\mathcal{H}}\right| \\
& =\log \left|\boldsymbol{I}_{N_{r}}+\frac{\rho}{N_{t}} \sum_{n=1, n \neq v}^{N_{t}} \tilde{\boldsymbol{h}}_{n} \tilde{\boldsymbol{h}}_{n}^{\mathcal{H}}\right|\left|\boldsymbol{I}_{N_{r}}+\left(\boldsymbol{I}_{N_{r}}+\frac{\rho}{N_{t}} \sum_{n=1, n \neq v}^{N_{t}} \tilde{\boldsymbol{h}}_{n} \tilde{\boldsymbol{h}}_{n}^{\mathcal{H}}\right)^{-1} \frac{\rho}{N_{t}} \tilde{\boldsymbol{h}}_{v} \tilde{\boldsymbol{h}}_{v}^{\mathcal{H}}\right| \\
& =\mathcal{C}_{N_{r}, N_{t}-1}^{[v]}+\log \left(1+\operatorname{SINR}_{N_{r}, N_{t}, v}\right),
\end{aligned}
$$

where $\mathcal{C}_{N_{r}, N_{t}-1}^{[v]}$ is the capacity of the $N_{r} \times\left(N_{t}-1\right)$ system without transmit antenna $v$. It follows from (26) that

$$
N_{t} \mathcal{C}_{N_{r}, N_{t}}=\sum_{\nu=1}^{N_{t}}\left[\mathcal{C}_{N_{r}, N_{t}-1}^{[v]}+\log \left(1+\operatorname{SINR}_{N_{r}, N_{t}, v}\right)\right] .
$$

On the other hand, from (8) and (25), we have the relation:

$$
\sum_{v=1}^{N_{t}} \log \left(1+\operatorname{SINR}_{N_{r}, N_{t}, v}\right) \leq \mathcal{C}_{N_{r}, N_{t}}
$$

where the equality holds if and only if $\operatorname{SINR}_{N_{r}, N_{t}, n}=\operatorname{SINR}_{n}^{S I C}$ for all $n \in\left[1, N_{t}\right]$, i.e., the channel matrix $\boldsymbol{H}$ is orthogonal. Combining (27) and (28) results in

$$
\mathcal{C}_{N_{r}, N_{t}} \leq \frac{1}{N_{t}-1} \sum_{v=1}^{N_{t}} \mathcal{C}_{N_{r}, N_{t}-1}^{[v]}
$$

Capacity per transmit antenna for $N_{r} \times N_{t}$ system can be defined as $\overline{\mathcal{C}}_{N_{r}, N_{t}}=\frac{1}{N_{t}} \mathcal{C}_{N_{r}, N_{t}}$. Similarly for $N_{r} \times\left(N_{t}-1\right)$ system, as $\overline{\mathcal{C}}_{N_{r}, N_{t}-1}^{[v]}=\frac{1}{N_{t}-1} \mathcal{C}_{N_{r}, N_{t}-1}^{[v]}$. Then, we obtain from (29) that

$$
\overline{\mathcal{C}}_{N_{r}, N_{t}}=\frac{1}{N_{t}} \mathcal{C}_{N_{r}, N_{t}} \leq \frac{1}{N_{t}} \sum_{v=1}^{N_{t}} \frac{1}{N_{t}-1} \mathcal{C}_{N_{r}, N_{t}-1}^{[v]}=\frac{1}{N_{t}} \sum_{v=1}^{N_{t}} \overline{\mathcal{C}}_{N_{r}, N_{t}-1}^{[v]} .
$$

This means that the capacity per transmit antenna of $N_{r} \times N_{t}$ system is in general smaller than the average capacity per transmit antenna of $N_{r} \times\left(N_{t}-1\right)$ system. The relation in Eq. (30) is satisfied for any channel (channel-independent) and for any SNR.

To get more insights, we assume that

\section{Assumption 2.}

$$
P\left(\boldsymbol{H}^{[1]}\right)=P\left(\boldsymbol{H}^{[2]}\right)=\cdots=P\left(\boldsymbol{H}^{\left[N_{t}\right]}\right),
$$

where $\boldsymbol{H}^{[v]}$ denotes the channel matrix when transmit antenna $v$ is dropped from the $N_{r} \times N_{t}$ system. 
Multiplying both sides of (30) by $P(\boldsymbol{H})$ and taking the average over $P(\boldsymbol{H})$, we can conclude that

Theorem 3. Suppose an $N_{r} \times N_{t}$ system where $N_{r} \geq N_{t} \geq 2$. Let the capacity per transmit antenna of $M \times N$ MIMO system be $\overline{\mathcal{C}}_{M, N}$. If the MIMO channel satisfies Assumption 2, then the mean capacity per transmit antenna is an decreasing function in the number of transmit antennas, i.e.,

$$
E\left\{\overline{\mathcal{C}}_{N_{r}, N_{t}}\right\}<E\left\{\overline{\mathcal{C}}_{N_{r}, N_{t}-1}\right\},
$$

where $N_{r} \times N_{t}$ system and $N_{r} \times\left(N_{t}-1\right)$ system have the same transmit power at each antenna.

Intuitively, this result may be quite reasonable, since in the original $N_{r} \times N_{t}$ system, symbols from transmit antenna $v$ can be considered as an interference to symbols from transmit antenna $n$ and the effect of symbols from transmit antenna $v$ is absent if transmit antenna $v$ is removed. For i.i.d. Rayleigh channels at high SNR, the diversity order of $N_{r} \times N_{t}$ systems is $N_{r}-N_{t}+1$ and hence reducing $N_{t}$ increases diversity order (Winters et al., 1994), while the capacity scales with $\min \left(N_{t}, N_{r}\right)$ (Tse and Viswanath, 2005). However, diversity or capacity gain at high SNR for Rayleigh channels does not imply capacity gain at all SNR for other channels. Thus, our result is not self-evident. From (32), we can find a fundamental tradeoff between bandwidth efficiency and capacity gain for any channel pdf at any value of SNR, i.e., if one increases the number of transmit antennas, then bandwidth efficiency or multiplexing gain is enhanced but the average capacity per transmit antenna is degraded.

Theorem 3 is in sharp contrast to Theorem 1 . The mean capacity per transmit antenna is an increasing function in the number of receive antennas, which is easily concluded from Theorem 1 , while the mean capacity per transmit antenna is a decreasing function in the number of transmit antennas.

\subsection{Block MMSE equalization case}

We return to block MMSE equalization case and will see that similar results for MMSE-SIC equalization also hold for block MMSE equalization.

Let us denote the (post-processing) SINR of symbol $n$ of the $N_{r} \times\left(N_{t}-1\right)$ system without transmit antenna $v$ after block MMSE equalization as $\operatorname{SINR}_{N_{r}, N_{t}-1, n}^{[v]}$. It has been shown in (Ohno and Teo, 2007) that for $n \neq v$,

$$
\operatorname{SINR}_{N_{r}, N_{t}-1, n}^{[v]} \geq \operatorname{SINR}_{N_{r}, N_{t}, n} .
$$

Hence, removing one transmit antenna, i.e., reducing the bandwidth efficiency, improves the SINR of each symbol transmitted from the remaining antennas and hence its information rate, i.e., if we denote the achievable information rate from antenna $n$ of $N_{r} \times N_{t}$ system and of $N_{r} \times\left(N_{t}-1\right)$ system respectively as $\mathcal{C}_{N_{r}, N_{t}, n}^{B}$ and $\mathcal{C}_{N_{r}, N_{t}-1, n^{\prime}}^{B,[v]}$, then

$$
\mathcal{C}_{N_{r}, N_{t}-1, n}^{B,[v]} \geq \mathcal{C}_{N_{r}, N_{t}, n}^{B} \text { for } n \neq v .
$$

The achievable information rate of $N_{r} \times N_{t}$ system per transmit antenna is expressed as

$$
\overline{\mathcal{C}}_{N_{r}, N_{t}}^{B}=\frac{1}{N_{t}} \sum_{n=1}^{N_{t}} \mathcal{C}_{N_{r}, N_{t}, n}^{B} .
$$


Similarly, the achievable information rate of $N_{r} \times\left(N_{t}-1\right)$ system without transmit antenna $v$ is

$$
\overline{\mathcal{C}}_{N_{r}, N_{t}-1}^{B,[v]}=\frac{1}{N_{t}-1} \sum_{n=1, n \neq v}^{N_{t}} \mathcal{C}_{N_{r}, N_{t}-1, n}^{B,[v]} .
$$

Subsequently, we have

$$
\begin{aligned}
\overline{\mathcal{C}}_{N_{r}, N_{t}}^{B}-\frac{1}{N_{t}} \sum_{\nu=1}^{N_{t}} \overline{\mathcal{C}}_{N_{r}, N_{t}-1}^{B,[v]} & =\frac{1}{N_{t}} \sum_{n=1}^{N_{t}} \mathcal{C}_{N_{r}, N_{t}, n}^{B}-\frac{1}{N_{t}} \sum_{v=1}^{N_{t}}\left(\frac{1}{N_{t}-1} \sum_{n=1, n \neq v}^{N_{t}} \mathcal{C}_{N_{r}, N_{t}-1, n}^{B,[v]}\right) \\
& =\frac{1}{N_{t}} \sum_{n=1}^{N_{t}}\left[\mathcal{C}_{N_{r}, N_{t}, n}^{B}-\left(\frac{1}{N_{t}-1} \sum_{v=1, v \neq n}^{N_{t}} \mathcal{C}_{N_{r}, N_{t}-1, n}^{B,[v]}\right)\right] .
\end{aligned}
$$

One finds from (34) that the argument in the brackets of (37) is less than or equal to 0 , which leads to

$$
\overline{\mathcal{C}}_{N_{r}, N_{t}}^{B} \leq \frac{1}{N_{t}} \sum_{v=1}^{N_{t}} \overline{\mathcal{C}}_{N_{r}, N_{t}-1}^{B,[v]} .
$$

This shows that if one transmit antenna is randomly removed with probability $1 / N_{t}$, for a fixed number of receive antennas, the achievable information rate per transmit antenna of the $N_{r} \times N_{t}$ system is never larger than the average achievable information rate per transmit antenna of $N_{r} \times\left(N_{t}-1\right)$ system. Since the equality sign holds only for some special cases, on the average, reducing the number of transmit antennas improves information rate per transmit antenna. We can again find a pure tradeoff between bandwidth efficiency and capacity gain.

If we average (38) with respect to channel pdf satisfying Assumption 2, we can state that:

Theorem 4. Suppose an $N_{r} \times N_{t}$ system with block MMSE equalization where $N_{r} \geq N_{t} \geq 2$. Let the achievable information rate per transmit antenna of $M \times N$ MIMO system be $\overline{\mathcal{C}}_{M, N}^{B}$. If the MIMO channel satisfies Assumption 2, then the average capacity per transmit antenna is a decreasing function in the number of transmit antennas, i.e.,

$$
E\left\{\overline{\mathcal{C}}_{N_{r}, N_{t}}^{B}\right\}<E\left\{\overline{\mathcal{C}}_{N_{r}, N_{t}-1}^{B}\right\},
$$

where $N_{r} \times N_{t}$ system and $N_{r} \times\left(N_{t}-1\right)$ system have the same transmit power per antenna.

\section{Numerical simulations}

To validate our theoretical findings, we perform computer simulations on the MIMO system for different antenna sizes. The results for both MMSE-SIC and block MMSE equalizations are presented. In our simulations, we always keep the average overall receive power of each symbol the same as in our theoretical analysis. We plot the information rate per transmit antenna with respect to $E_{b} / N_{0}$ where at each $E_{b} / N_{0}$, the average receive power of each symbol is kept constant regardless of the antenna configuration.

In simulations 1 and 2, we see the effect of the number of receive antennas on the information rate averaged with respect to random receive antenna dropping over a fixed channel. Fig. 1 and Fig. 2 illustrate the results for a fixed $N_{t}=4$ and $N_{r}$ varying from 8 to 4 for MMSE-SIC equalization (which achieves the capacity) and block MMSE equalization, respectively. As 
one may see from these figures, the information rate averaged with respect to random receive antenna dropping degrades with a decrease in $N_{r}$. Especially for block MMSE equalization, the degradation of the information rate is significant when $N_{r}$ is decreased. These results hold true not just for this fixed channel but also for other channels we have tested, which confirm the fidelity of (15) and (23), i.e., our analysis of the ordering of the information rate based on concavity of the log det is correct.

Then, in the next two simulations, we test the impact of the number of transmit antennas on the information rate averaged with respect to random transmit antenna dropping over a fixed channel. We set $N_{r}=8$ and decrease $N_{t}$ from 8 to 4 . The simulation results are shown in Fig. 3 and Fig. 4 for MMSE-SIC equalization and block MMSE equalization respectively. We observe that as the number of transmit antennas is reduced, the information rate averaged with respect to random transmit antenna dropping improves even when the transmit power of each transmit antenna remains the same. The information rate increase for MMSE-SIC equalization is small but is quite significant for block MMSE equalization when we decrease the number of transmit antennas. Evidently, this ordered information rate performances validate (30) and (38).

In our subsequent simulations, instead of simulating over a fixed channel, we take the average over $10^{5}$ Rice channels of Rice factor 2 that compose of zero mean Gaussian taps with unit variance in order to verify the effect of the number of receive antennas on the information rate per transmit antenna averaged over random channels. Fig. 5 and Fig. 6 depict the results for a fixed $N_{t}=4$ and $N_{r}$ varying from 8 to 4 for MMSE-SIC and block MMSE equalizations respectively. We can see that the information rate averaged over random channels degrade with a decrease in $N_{r}$ for both MMSE-SIC and block MMSE equalizations, demonstrating that the information rate averaged over random channels is an increasing function in the number of receive antennas. These are in good agreement with Theorem 1 and Theorem 2, which hold true for all SNR.

Lastly, to see the effect of the number of transmit antennas on the information rate per transmit antenna averaged over random channels, Fig. 7 and Fig. 8 show the results for a fixed $N_{r}=8$ and $N_{t}$ varying from 8 to 4 for MMSE-SIC and block MMSE equalizations respectively. The simulation results confirm Theorem 3 and Theorem 4 as the information rate averaged over random channels improves with a decrease in $N_{t}$ (or equivalently, decrease in both bandwidth efficiency and multiplexing gain). In other words, the information rate averaged over random channels is a decreasing function in the number of transmit antennas. These ordered performances show unequivocally that there is an undisputed tradeoff between the information rate and bandwidth efficiency (and/or multiplexing gain).

\section{Conclusions}

Based on our novel point of view, we have demonstrated theoretically that under the condition of a fixed overall received power and a fixed number of transmit antennas, the information rate averaged over random receive antenna dropping and the information rate averaged over random channels degrade with a decrease in the number of receive antennas. These results are derived from the basic property of the log det function. On the other hand, for a fixed number of receive antennas, we have proven that a decrease in the number of transmit antennas translates into an amelioration in both the information rate averaged over random transmit antenna dropping as well as the information rate averaged over random channels, 


\section{MMSE SIC}

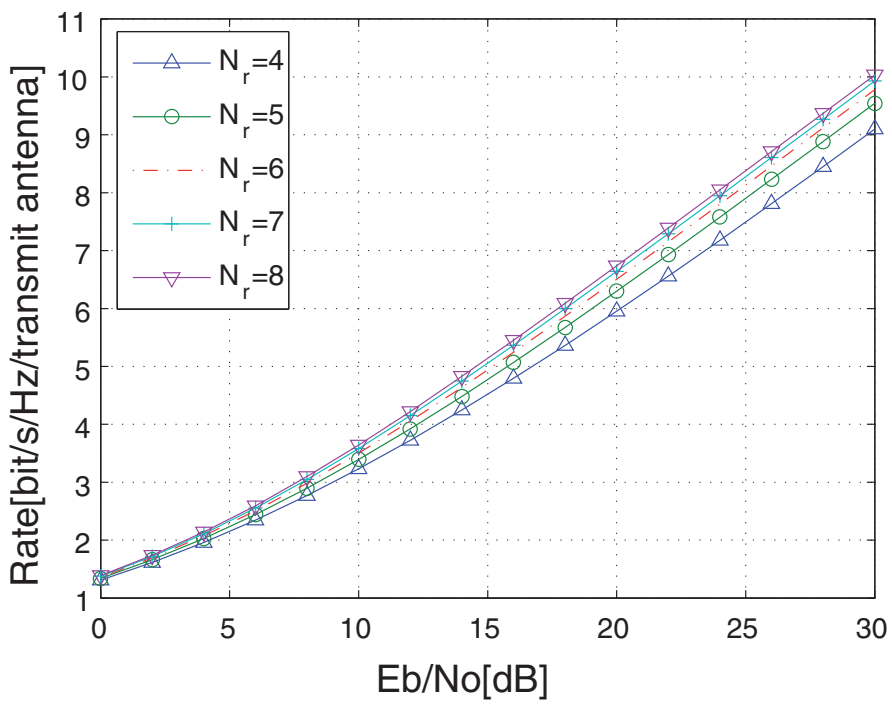

Fig. 1. Information rate per transmit antenna with respect to random receive antenna dropping for MMSE-SIC over a fixed channel for a fixed $N_{t}=4$ and varying $N_{r}$.

\section{Block MMSE}

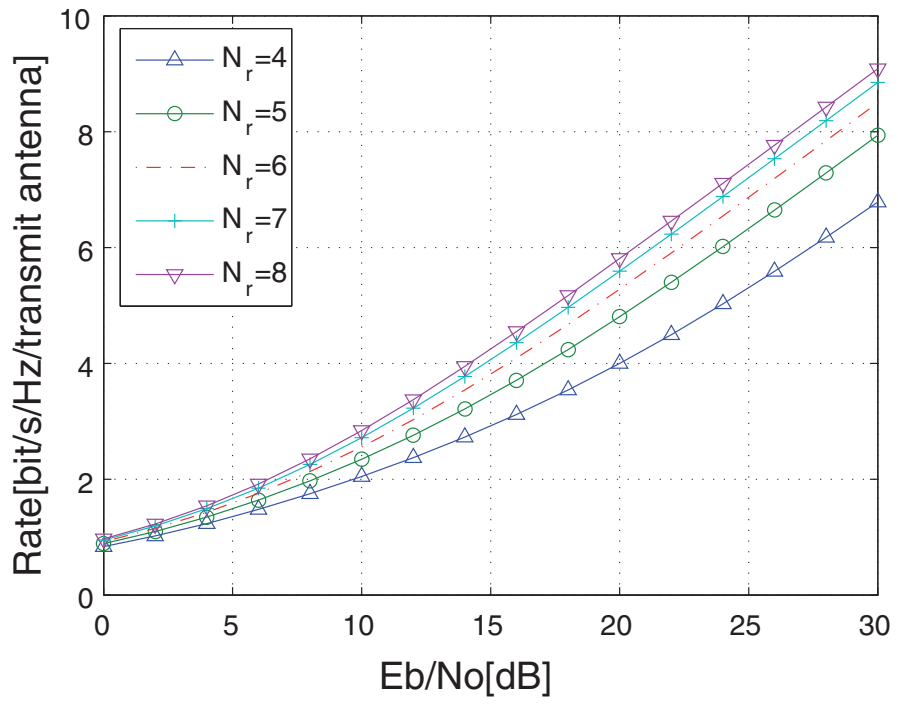

Fig. 2. Information rate per transmit antenna with respect to random receive antenna dropping for block MMSE over a fixed channel for a fixed $N_{t}=4$ and varying $N_{r}$. 


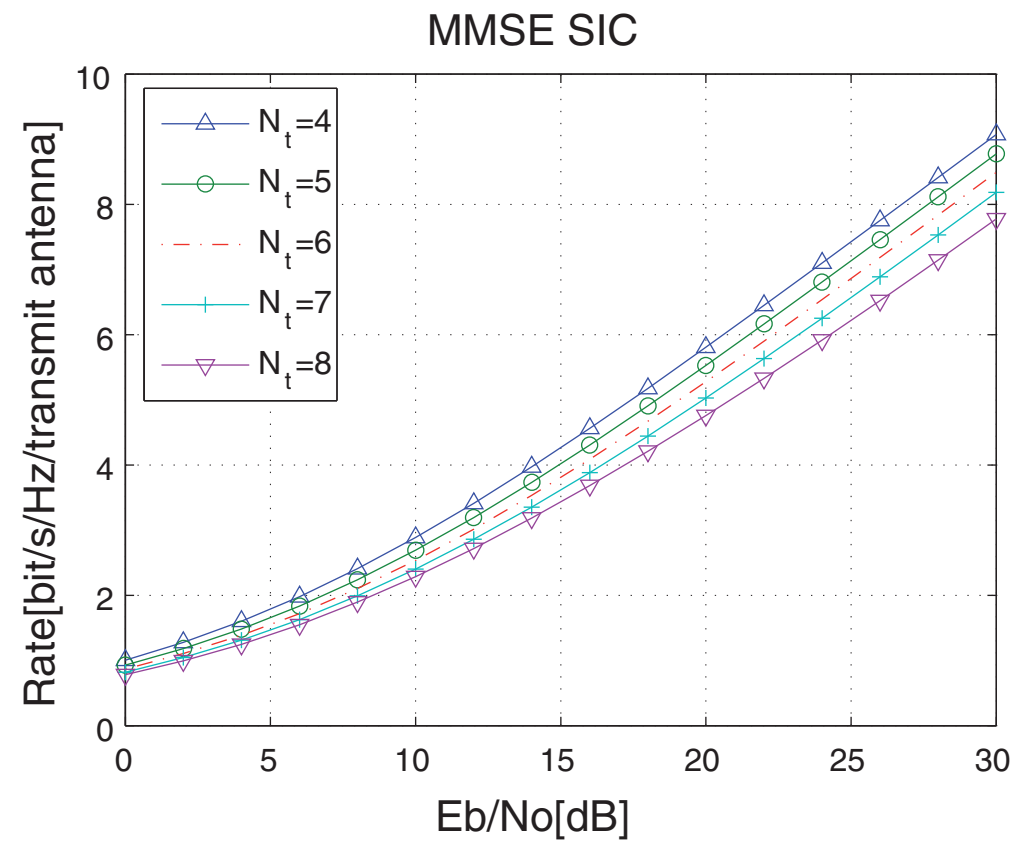

Fig. 3. Information rate per transmit antenna with respect to random transmit antenna dropping for MMSE-SIC over a fixed channel for a fixed $N_{r}=8$ and varying $N_{t}$.

\section{Block MMSE}

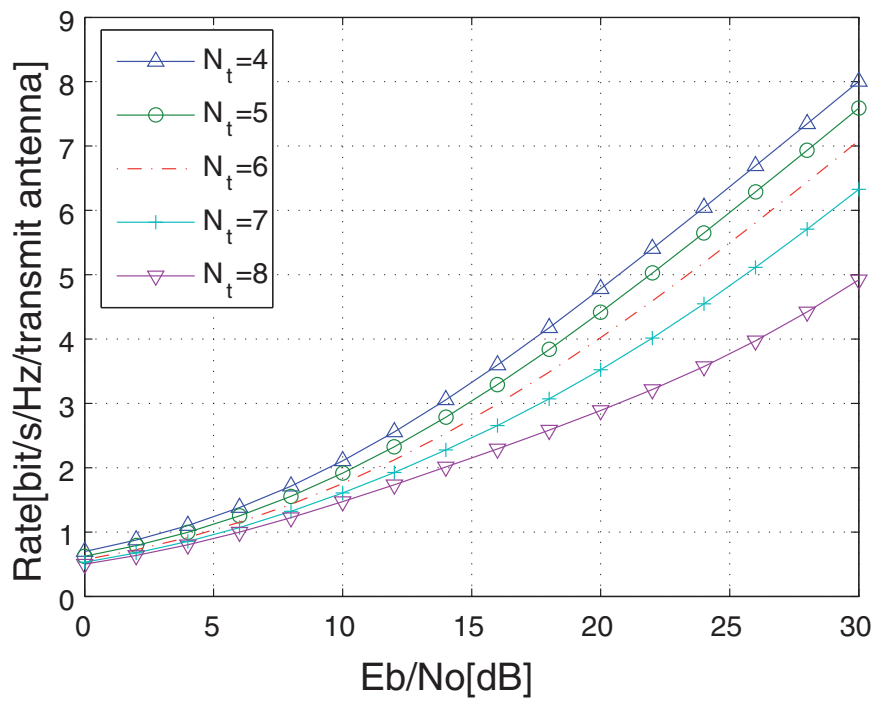

Fig. 4. Information rate per transmit antenna with respect to random transmit antenna dropping for block MMSE over a fixed channel for a fixed $N_{r}=8$ and varying $N_{t}$. 


\section{MMSE SIC}

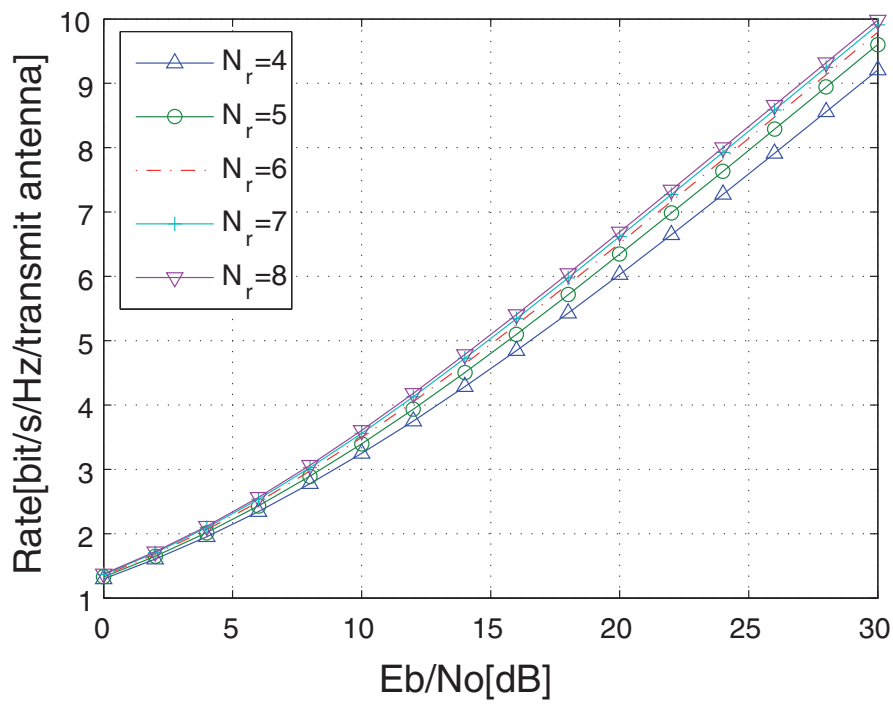

Fig. 5. Information rate per transmit antenna averaged over random channels for MMSE-SIC for a fixed $N_{t}=4$ and varying $N_{r}$.

\section{Block MMSE}

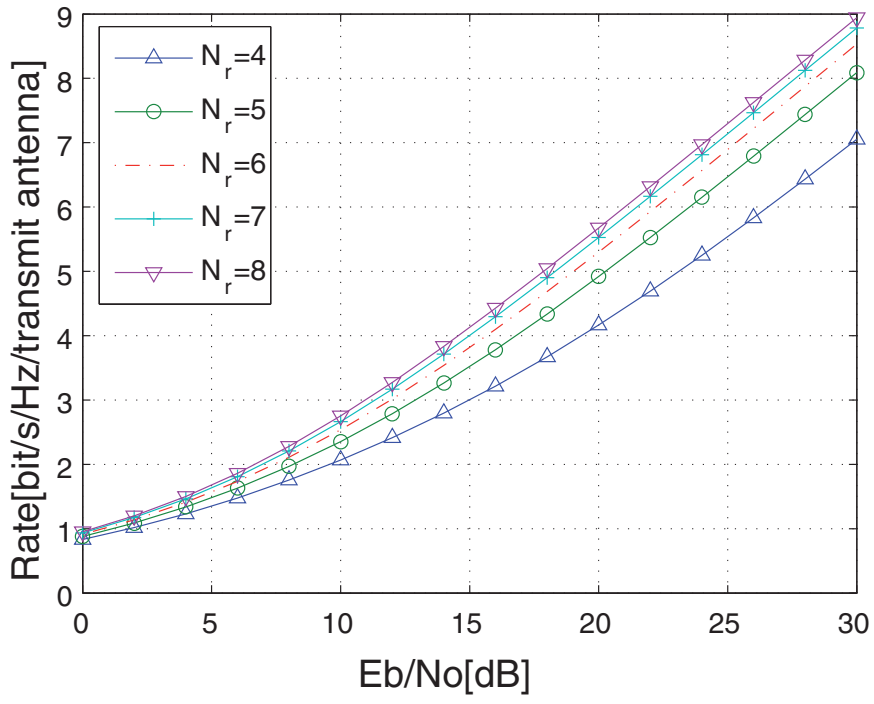

Fig. 6. Information rate per transmit antenna averaged over random channels for block MMSE for a fixed $N_{t}=4$ and varying $N_{r}$. 
MMSE SIC

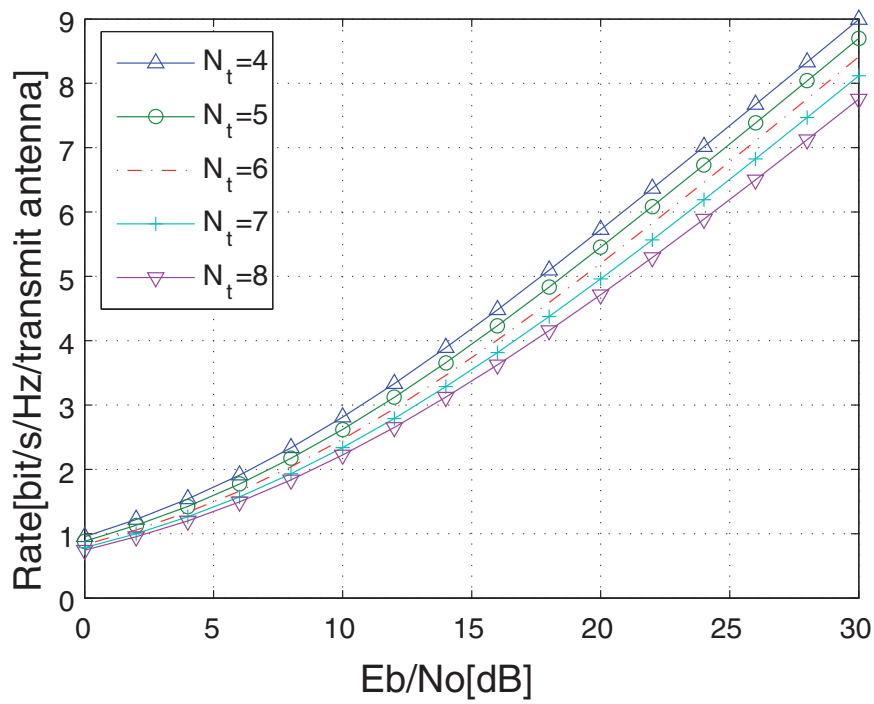

Fig. 7. Information rate per transmit antenna averaged over random channels for MMSE-SIC for a fixed $N_{r}=8$ and varying $N_{t}$.

\section{Block MMSE}

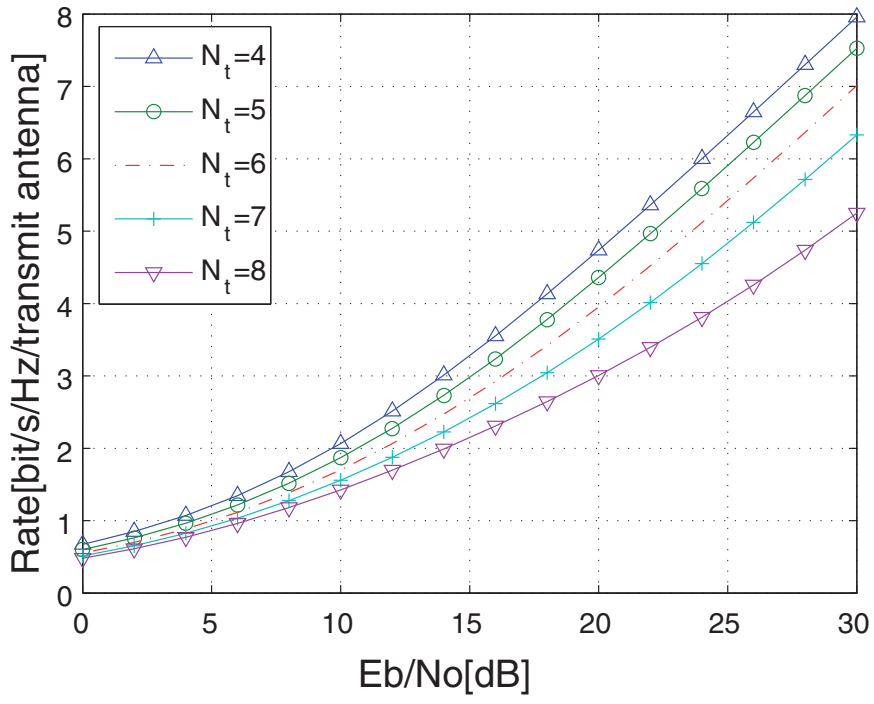

Fig. 8. Information rate per transmit antenna averaged over random channels for block MMSE for a fixed $N_{r}=8$ and varying $N_{t}$. 
which highlight the tradeoff between capacity and bandwidth efficiency (and multiplexing gain). All these results hold for any kind of i.i.d. channel regardless of the channel pdf and is valid at any SNR. Numerical simulations corroborated our analysis.

\section{References}

Alamouti, S. M. (1998). A simple transmit diversity for wireless communications, IEEE Journal on Selected Areas in Communications 16(8): 1451-1458.

Caire, G. and Shamai, S. (1999). On the capacity of some channels with channel state information, IEEE Transactions on Information Theory pp. 2007-2019.

Catreux, S., Greenstein, L. J. and Erceg, V. (2003). Some results and insights on the performance gains of MIMO systems, IEEE Journal on Selected Areas in Communications 21(5): 839-847.

Chiani, M., Win, M. Z. and Zanella, A. (2003). On the capacity of spatially correlated MIMO rayleigh-fading channels, IEEE Transactions on Information Theory 49(10): 2363-2371.

Foschini, G. J. (1996). Layered space-time architecture for wireless communication in a fading environment when using multi-element antennas, Bell Labs Tech. J. 1(2): 41-59.

Foschini, G. J., Golden, G., Valenzuela, R. and Wolniansky, P. (1999). Simplified processing for high spectral efficiency wireless communication employing multi-element arrays, IEEE Journal on Selected Areas in Communications 17(11): 1841-1852.

Goldsmith, A., Jafar, S. A., Jindal, N. and Vishwanath, S. (2003). Capacity limits of MIMO channels, IEEE Journal on Selected Areas in Communications 21(5): 684-702.

Kay, S. M. (1993). Fundamentals of Statistical Signal Processing, Vol. 1, Prentice Hall.

Larsson, E. G. and Stoica, P. (2003). Space-time block coding for wireless communications, Cambridge University Press.

Ma, X. and Giannakis, G. B. (2003). Full-diversity full-rate complex-field space-time coding, IEEE Transactions on Signal Processing 51(11): 2917-2930.

Marzetta, T. L. and Hochwald, B. M. (1999). Capacity of a mobile multiple-antenna communication link in rayleigh flat fading, IEEE Transactions on Information Theory 45(1): 139-157.

Narasimhan, R. (2003). Spatial multiplexing with transmit antenna and constellation selection for correlated MIMO fading channels, IEEE Transactions on Signal Processing 51(11): 2829-2838.

Ohno, S. and Teo, K. A. D. (2007). Universal BER performance ordering of MIMO systems over flat channels, IEEE Transactions on Wireless Communications 6(10): 3678-3687.

Smith, P. J., Roy, S. and Shafi, M. (2003). Capacity of MIMO systems with semicorrelated flat fading, IEEE Transactions on Information Theory 49(10): 2781-2788.

Tarokh, V., Jafarkhani, H. and Calderbank, A. R. (1999). Space-time block coding for wireless communications: performance results, IEEE Trans. Communication 17(3): 451-460.

Telatar, I. E. (1999). Capacity of multiple-antenna Gaussian channels, Eur. Trans. Tel. pp. 585-595.

Tse, D. and Viswanath, P. (2005). Fundamentals of wireless communication, Cambridge University Press.

Winters, J. H., Salz, J. and Gitlin, R. D. (1994). The impact of antenna diversity on the capacity of wireless communication systems, IEEE Transactions on Communications 42(234): 1740-1751. 
Xin, Y., Wang, Z. and Giannakis, G. B. (2003). Space-time diversity systems based on linear constellation precoding, IEEE Transactions on Wireless Communications 2(2): 294-309.

Zheng, L. and Tse, D. N. C. (2003). Diversity and multiplexing: a fundamental tradeoff in multiple-antenna channels, IEEE Transactions on Information Theory 49(5): 1073-1096. 


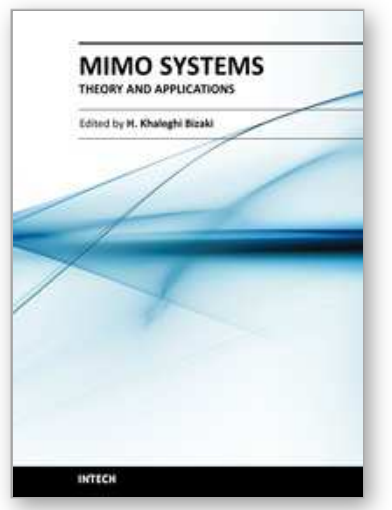

\author{
MIMO Systems, Theory and Applications \\ Edited by Dr. Hossein Khaleghi Bizaki
}

ISBN 978-953-307-245-6

Hard cover, 488 pages

Publisher InTech

Published online 04, April, 2011

Published in print edition April, 2011

In recent years, it was realized that the MIMO communication systems seems to be inevitable in accelerated evolution of high data rates applications due to their potential to dramatically increase the spectral efficiency and simultaneously sending individual information to the corresponding users in wireless systems. This book, intends to provide highlights of the current research topics in the field of MIMO system, to offer a snapshot of the recent advances and major issues faced today by the researchers in the MIMO related areas. The book is written by specialists working in universities and research centers all over the world to cover the fundamental principles and main advanced topics on high data rates wireless communications systems over MIMO channels. Moreover, the book has the advantage of providing a collection of applications that are completely independent and self-contained; thus, the interested reader can choose any chapter and skip to another without losing continuity.

\title{
How to reference
}

In order to correctly reference this scholarly work, feel free to copy and paste the following:

Shuichi Ohno and Kok Ann Donny Teo (2011). Another Interpretation of Diversity Gain of MIMO Systems, MIMO Systems, Theory and Applications, Dr. Hossein Khaleghi Bizaki (Ed.), ISBN: 978-953-307-245-6, InTech, Available from: http://www.intechopen.com/books/mimo-systems-theory-and-applications/anotherinterpretation-of-diversity-gain-of-mimo-systems

\section{INTECH}

open science | open minds

\section{InTech Europe}

University Campus STeP Ri

Slavka Krautzeka 83/A

51000 Rijeka, Croatia

Phone: +385 (51) 770447

Fax: +385 (51) 686166

www.intechopen.com

\section{InTech China}

Unit 405, Office Block, Hotel Equatorial Shanghai

No.65, Yan An Road (West), Shanghai, 200040, China 中国上海市延安西路65号上海国际贵都大饭店办公楼 405 单元 Phone: +86-21-62489820

Fax: $+86-21-62489821$ 
(C) 2011 The Author(s). Licensee IntechOpen. This chapter is distributed under the terms of the Creative Commons Attribution-NonCommercialShareAlike-3.0 License, which permits use, distribution and reproduction for non-commercial purposes, provided the original is properly cited and derivative works building on this content are distributed under the same license. 